\title{
Synchronous Property-Key Fact on Quantum Interferences
}

\section{Particle Simulation on Double Path Experiment}

\author{
Jeffrey Zheng
}

\begin{abstract}
Double-slit experiment plays a key role in Quantum Theory to distinct particle and wave interactions according to Feynman's claims. In this chapter, double path model and variant logic principle are applied to establish a simulation system for exhaustive testing targets. Using Einstein quanta interaction, different measure quaternion structures are investigated. Under Symmetry/Anti-symmetry and Synchronous/Asynchronous interaction conditions, eight groups of statistical results are generated as eight histograms to show their distributions. From this set of simulation results, it can be recognized that the synchronous condition is the key fact to generate quantum wave interference patterns and, in addition, the asynchronous condition is the key fact to make classic particle distributions. Sample results are illustrated and explanations are discussed.
\end{abstract}

Keywords Double path $\cdot$ Interaction $\cdot$ Probability $\cdot$ Statistics $\cdot$ Simulation

\section{Introduction}

Feynman explored quantum measurement puzzles deeply [1, 2] and emphasized: "The entire mystery of quantum mechanics is in the double-slit experiment." This experiment directly illustrates both classical and quantum interactive results. Under single and double slit conditions, dual visual distributions are shown in particle and wave statistical distributions linked to von Neumann's measure theory [3].

This work was supported by the Key Project on Electric Information and Next Generation IT Technology of Yunnan (2018ZI002), and Yunnan Advanced Overseas Scholar Project.

J. Zheng $(\varangle)$

Key Laboratory of Quantum Information of Yunnan, Yunnan University,

Kunming, China

e-mail: conjugatelogic@yahoo.com

J. Zheng

Key Laboratory of Software Engineering of Yunnan, Yunnan University, Kunming, China

(C) The Author(s) 2019

J. Zheng (ed.), Variant Construction from Theoretical Foundation to Applications,

https://doi.org/10.1007/978-981-13-2282-2_16 
From the 1970s, piloted by CHSH [4], Aspect used experiments to test Bell inequalities [5-7]. After 40 years of development, many accurate experiments [8-10] have been performed successfully worldwide using Laser, NMRI, large molecular, quantum coding, and quantum communication approaches [5-8, 11-26].

In this chapter, a double path model is established using the Mach-Zehnder interferometer. Different approaches of quantum measures: Einstein, $\mathrm{CHSH}$, and Aspect are investigated by quaternion structures. Under multiple-variable logic functions and variant principle, logic functions can be transferred into variant logic expression as variant measures. Under such conditions, a variant simulation model is proposed. A given logic function $f$ can be represented as two meta-logic functions $f_{+}$and $f_{-}$to simulate single and double path conditions. $N$ bits of input vectors are exhausted by $2^{N}$ states for measured data, recursive data are organized into eight histograms. Results are determined by symmetry/anti-symmetry properties evident in these histograms. Both results are obtained consistently from this model on synchronous/asynchronous conditions. Based on this set of simulation results, synchronous condition shows significant relationship linked to interference properties.

\section{Double Path Model and Their Measures}

\subsection{Mach-Zehnder Interferometer Model}

The Mach-Zehnder interferometer is the most popular device [6, 20] to support Young's double-slit experiment.

In Fig. 1a, a double path interferometer is shown. An input signal $X$ under control function $f$ causes Laser LS to emit the output signal $\rho$ under BP (Bi-polarized filter) operation output a pair of signals: $\rho^{+}$and $\rho^{-}$. Both signals are processed by SW output $\rho_{L}^{+}$and $\rho_{R}^{-}$, and then IM to generate output signals $\operatorname{IM}\left(\rho_{L}^{+}, \rho_{R}^{-}\right)$. In Fig. 1b, a representation model has been described with the same signals being used.

(a)

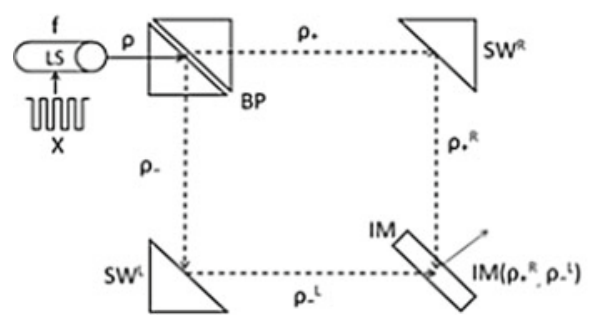

(b)

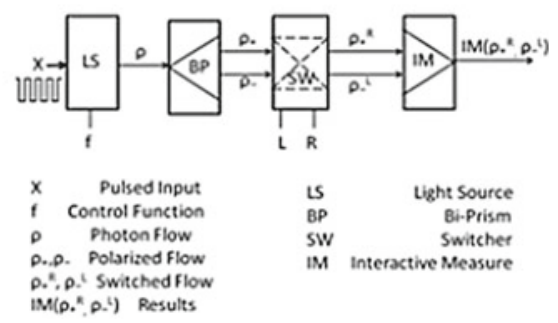

Fig. 1 Double path model a Mach-Zehnder double path model, b Description model 


\subsection{Emission and Absorption Measures of Quantum Interaction}

Einstein established a model to describe atomic interaction [27-30] with radiation in 1916. For two-state systems, let a system have two energy states: the ground state $E_{1}$ and the excited state $E_{2}$. Let $N_{1}$ and $N_{2}$ be the average numbers of atoms in the ground and excited states, respectively. The numbers of states are changed from an emission state $E_{2}$ to $E_{1}$ with a rate $\frac{\mathrm{d} N_{21}}{\mathrm{~d} t}$, in the same time; the numbers of ground states are determined by absorbed energies from $E_{1}$ to $E_{2}$ with a rate $\frac{\mathrm{d} N_{12}}{\mathrm{~d} t}$, respectively. Let $N_{12}$ be the number of atoms from $E_{1}$ to $E_{2}$ and $N_{21}$ be the numbers from $E_{2}$ to $E_{1}$. In Einstein's model, a measurement quaternion is $\left\langle N_{1}, N_{2}, N_{12}, N_{21}\right\rangle$.

$\mathrm{CHSH}$ proposed spin measures testing Bell inequalities $[4,6]$. They applied $\perp \rightarrow$ + and $\| \rightarrow-$ to establish a measurement quaternion

$$
\left\langle N_{++}(a, b), N_{+-}(a, b), N_{-+}(a, b), N_{--}(a, b)\right\rangle .
$$

Experimental testing of Bell inequalities was performed by Aspect [5] in 1982. Four parameters are measured: transmission rate $N_{t}$, reflection rate $N_{r}$, correspondent rate $N_{c}$, and the total number $N_{\omega}$ in $\omega$ time period. This set of measures is a quaternion $\left\langle N_{t}, N_{r}, N_{c}, N_{\omega}\right\rangle$. Among these, $N_{c}$ is a new data type not in Einstein and CHSH methods, this parameter could be an extension of synchronous/asynchronous timemeasurement.

\section{Simulation Systems}

\subsection{Simulation Model}

Using variant principle described in the next subsections, a $N$ bit $0-1$ vector $X$ and a given logic function $f$, all $N$ bit vectors are exhausted, variant measures generate two groups of histograms. This variant simulation system is composed of three components: Pre-process, Interaction, and Post-process, respectively, and shown in Fig. 2.

In Fig. 2a, three components of the variant simulation model are presented. At the pre-process, a $N$ bit $0-1$ vector $\mathrm{X}$ and a function $f$ feed in to output a signal $\rho$. After interactive component process, two groups of signals are the output: $u$ for symmetry group and $v$ for anti-symmetry group. In the post-process, all $N$ bit vectors are processed by pre-processing and interactive components until all of the $2^{N}$ data set has been processed to transform symmetry and anti-symmetry signals into eight histograms: four for symmetry distributions and another four for anti-symmetry distributions. 
Fig. 2 Variant simulation system; a Variant simulation system; b interactive component

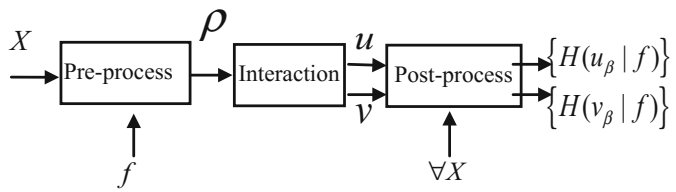

(a) Variant Simulation System

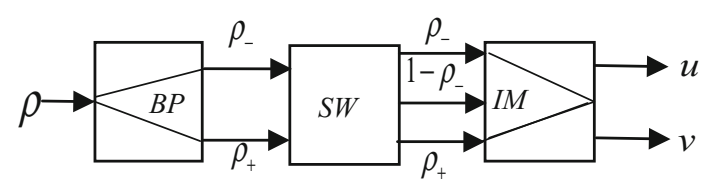

(b) Interaction Component

In Fig. 2b, only the interaction component is selected, input signal $\rho$ processed by BP to generate two signals $\left\{\rho_{-}, \rho_{+}\right\}$. SW output triple signals $\left\{\rho_{-}, 1-\rho_{-}, \rho_{+}\right\}$ though IM to generate two groups of signals $u$ and $v$.

\subsection{Variant Principle}

The variant principle is based on n-variable logic functions [31-33]. For any $n$ variables, $x=x_{n-1} \ldots x_{i} \ldots x_{0}, 0 \leq i<n, x_{i} \in\{0,1\}=B_{2}$. Let a position $j$ be the selected bit $0 \leq j<n, x_{j} \in B_{2}$ be the selected variable. Let output variable $\mathrm{y}$ and $n$-variable function $f, y=f(x), y \in B_{2}, x \in B_{2}^{n}$. For all states of $x$, a set $S(n)$ composed of the $2^{n}$ states can be divided into two sets: $S_{0}^{j}(n)$ and $S_{1}^{j}(n)$.

$$
\left\{\begin{array}{l}
S_{0}^{j}(n)=\left\{x \mid x_{j}=0, \forall x \in B_{2}^{n}\right\} \\
S_{1}^{j}(n)=\left\{x \mid x_{j}=1, \forall x \in B_{2}^{n}\right\} \\
S(n)=\left\{S_{0}^{j}(n), S_{1}^{j}(n)\right\}
\end{array}\right.
$$

For a given logic function $f$, there are input and output pair relationships to define four meta-logic functions $\left\{f_{\perp}, f_{+}, f_{-}, f_{T}\right\}$ :

$$
\left\{\begin{array}{l}
f_{\perp}(x)=\left\{f(x) \mid x \in S_{0}^{j}(n), y=0\right\} \\
f_{+}(x)=\left\{f(x) \mid x \in S_{0}^{j}(n), y=1\right\} \\
f_{-}(x)=\left\{f(x) \mid x \in S_{1}^{j}(n), y=0\right\} \\
f_{T}(x)=\left\{f(x) \mid x \in S_{1}^{j}(n), y=1\right\}
\end{array}\right.
$$


Two logic canonical expressions: AND-OR form is selected by $\left\{f_{+}(x), f_{T}(x)\right\}$ as $\mathrm{y}=1$ items, and OR-AND form is selected from $\left\{f_{-}(x), f_{\perp}(x)\right\}$ as $\mathrm{y}=0$ items. Considering $\left\{f_{T}(x), f_{\perp}(x)\right\}, x_{j}=y$ items, they are invariant themselves.

To select $\left\{f_{+}(x), f_{-}(x)\right\} ; x_{j} \neq y$ forming variant logic expression. Let $f(x)=$ $\left\langle f_{+}|x| f_{-}\right\rangle$be a variant logic expression. Any logic function can be expressed as a variant logic form. In $\left\langle f_{+}|x| f_{-}\right\rangle$structure, $f_{+}$selected 1 item in $S_{0}^{j}(n)$ as same as the AND-OR standard expression, and $f_{-}$selecting relevant parts as same as the OR-AND expression 0 items in $S_{1}^{j}(n)$. For a convenient understanding of variant representation, two-variable logic structures are illustrated for its 16 functions shown in Table 1.

For example, checking two functions $f=3$ and $f=12$ :

$$
\begin{aligned}
& \left\{f=3:=\langle 0 \mid 3\rangle, f_{+}=11:=\langle 0 \mid \phi\rangle, f_{-}=2:=\langle\phi \mid 3\rangle\right\} \\
& \left\{f=12:=\langle 2 \mid 1\rangle, f_{+}=14:=\langle 2 \mid \phi\rangle, f_{-}=8:=\langle\phi \mid 1\rangle\right\}
\end{aligned}
$$

\subsection{Variant Measures}

Let $\Delta$ be variant measure function $[23,33]$.

$$
\begin{gathered}
\Delta=\left\langle\Delta_{\perp}, \Delta_{+}, \Delta_{-}, \Delta_{T}\right\rangle \\
\Delta f(x)=\left\langle\Delta_{\perp} f(x), \Delta_{+} f(x), \Delta_{-} f(x), \Delta_{T} f(x)\right\rangle \\
=\left\langle\Delta f_{\perp}(x), \Delta f_{+}(x), \Delta f_{-}(x), \Delta f_{T}(x)\right\rangle \\
\Delta f_{\alpha}(x)=\left\{\begin{array}{l}
1, \text { if } f(x)=f_{\alpha}(x), \alpha \in\{\perp,+,-\mathrm{T}\} \\
0, \text { others }
\end{array}\right.
\end{gathered}
$$

For any given n-variable state there is one position in $\Delta f(x)$ to be 1 and other three positions are 0 .

For any $N$ bit $0-1$ vector $X ; X=X_{N-1} \ldots X_{J} \ldots X_{0}, 0 \leq J<N, X_{\mathrm{J}} \in \beta_{2}, X \in$ $\beta_{2}^{N}$ under n-variable function $f$, n bit $0-1$ output vector $Y, Y=f(X)=\left\langle f_{+}|X| f_{-}\right\rangle$, $Y=Y_{N-1} \ldots Y_{\mathrm{J}} \ldots Y_{0}, 0 \leq \mathrm{J}<N, Y_{\mathrm{j}} \in \beta_{2}, Y \in \beta_{2}^{N}$.

For the $J$ th position, be $x^{\mathrm{J}}=\left[\ldots X_{\mathrm{J}} \ldots\right] \in \beta_{2}^{n}$ to form $Y_{\mathrm{J}}=f\left(x^{\mathrm{J}}\right)=\left\langle f_{+}\left|x^{\mathrm{J}}\right| f_{-}\right\rangle$, let $N$ bit positions be cyclic linked. Variant measures of $f(X)$ can be decomposed

$$
\Delta\langle X: Y\rangle=\Delta f(X)=\sum_{J=0}^{N-1} \Delta f\left(x^{J}\right)=\left\langle N_{\perp}, N_{+}, N_{-}, N_{T}\right\rangle
$$

as a quaternion $\left\langle N_{\perp}, N_{+}, N_{-}, N_{T}\right\rangle$.

For example, $N=10$, given $f, Y=f(\mathrm{X})$. 
Table 1 Two variable logic functions and variable logic representation $(n=2, j=0)$

\begin{tabular}{|c|c|c|c|c|c|c|c|c|c|c|c|}
\hline $\begin{array}{c}f \\
\text { No. }\end{array}$ & $\begin{array}{l}f \in \\
S(2)\end{array}$ & $\begin{array}{c}3 \\
11\end{array}$ & $\begin{array}{c}2 \\
10\end{array}$ & $\begin{array}{c}1 \\
01\end{array}$ & $\begin{array}{c}0 \\
00\end{array}$ & $\begin{array}{l}f_{+} \in \\
S_{0}^{0}(2)\end{array}$ & $\begin{array}{c}3^{0} \\
11^{0}\end{array}$ & $\begin{array}{c}2^{1} \\
10^{1}\end{array}$ & $\begin{array}{c}1^{0} \\
01^{0}\end{array}$ & $\begin{array}{c}0^{1} \\
00^{1}\end{array}$ & $\begin{array}{l}f_{-} \in \\
S_{1}^{0}(2)\end{array}$ \\
\hline 0 & $\{\varnothing\}$ & 0 & 0 & 0 & 0 & $\langle\varnothing|$ & 1 & 0 & 1 & 0 & $|3,1\rangle$ \\
\hline 1 & $\{0\}$ & 0 & 0 & 0 & 1 & $\langle 0|$ & 1 & 0 & 1 & 1 & $|3,1\rangle$ \\
\hline 2 & $\{1\}$ & 0 & 0 & 1 & 0 & $\langle\varnothing|$ & 1 & 0 & 0 & 0 & $|3\rangle$ \\
\hline 3 & $\{1,0\}$ & 0 & 0 & 1 & 1 & $\langle 0|$ & 1 & 0 & 0 & 1 & $|3\rangle$ \\
\hline 4 & $\{2\}$ & 0 & 1 & 0 & 0 & $\langle 2|$ & 1 & 1 & 1 & 0 & $|3,1\rangle$ \\
\hline 5 & $\{2,0\}$ & 0 & 1 & 0 & 1 & $\langle 2,0|$ & 1 & 1 & 1 & 1 & $|3,1\rangle$ \\
\hline 6 & $\{2,1\}$ & 0 & 1 & 1 & 0 & $\langle 2|$ & 1 & 1 & 0 & 0 & $|3\rangle$ \\
\hline 7 & $\{2,1,0\}$ & 0 & 1 & 1 & 1 & $\langle 2,0|$ & 1 & 1 & 0 & 1 & $|3\rangle$ \\
\hline 8 & $\{3\}$ & 1 & 0 & 0 & 0 & $\langle\varnothing|$ & 0 & 0 & 1 & 0 & $|1\rangle$ \\
\hline 9 & $\{3,0\}$ & 1 & 0 & 0 & 1 & $\langle 0|$ & 0 & 0 & 1 & 1 & $|1\rangle$ \\
\hline 10 & $\{3,1\}$ & 1 & 0 & 1 & 0 & $\langle\varnothing|$ & 0 & 0 & 0 & 0 & $|\varnothing\rangle$ \\
\hline 11 & $\{3,1,0\}$ & 1 & 0 & 1 & 1 & $\langle 0|$ & 0 & 0 & 0 & 1 & $|\varnothing\rangle$ \\
\hline 12 & $\{3,2\}$ & 1 & 1 & 0 & 0 & $\langle 2|$ & 0 & 1 & 1 & 0 & $|1\rangle$ \\
\hline 13 & $\{3,2,0\}$ & 1 & 1 & 0 & 1 & $\langle 2,0|$ & 0 & 1 & 1 & 1 & $|1\rangle$ \\
\hline 14 & $\{3,2,1\}$ & 1 & 1 & 1 & 0 & $\langle 2|$ & 0 & 1 & 0 & 0 & $|\varnothing\rangle$ \\
\hline 15 & $\{3,2,1,0\}$ & 1 & 1 & 1 & 1 & $\langle 2,0|$ & 0 & 1 & 0 & 1 & $|\varnothing\rangle$ \\
\hline
\end{tabular}




$\begin{array}{llllllllllll}\mathrm{X} & = & 0 & 1 & 1 & 0 & 0 & 1 & 1 & 1 & 0 & 0 \\ \mathrm{Y} & = & 1 & 0 & 1 & 0 & 1 & 0 & 1 & 0 & 1 & 0 \\ \Delta(\mathrm{X}: \mathrm{Y}) & = & + & - & \mathrm{T} & \perp & + & - & \mathrm{T} & - & + & \perp\end{array}$

$$
\Delta f(X)=\left\langle N_{\perp}, N_{+}, N_{-}, N_{T}\right\rangle=\langle 2,3,3,2\rangle, N=10
$$

Input and output pairs are 0-1 variables on the four combinations. For any given function $f$, the quantitative relationship of $\{\perp,+,-, T\}$ is determined directly from input/output sequences.

\subsection{Measurement Equations}

Using variant quaternion, signals are calculated by following equations. For any $N$ bit 0-1 vector $X$, function $f$, under $\Delta$ measurement: $\Delta f(x)=\left\langle N_{\perp}, N_{+}, N_{-}, N_{T}\right\rangle$, $N=N_{\perp}+N_{+}+N_{-}+N_{T}$ Signal $\rho$ is defined by

$$
\begin{gathered}
\rho=\frac{\Delta f(x)}{N}=\left\langle\rho_{\perp}, \rho_{+}, \rho_{-}, \rho_{T}\right\rangle \\
\rho_{\alpha}=\frac{N_{\alpha}}{N}, \quad 0 \leq \rho_{\alpha} \leq 1, \quad \alpha \in\{\perp,+,-, T\}
\end{gathered}
$$

Using $\left\{\rho_{+}, \rho_{-}\right\}$, a pair of signals $\{u, v\}$ are formulated:

$$
\begin{gathered}
\left\{\begin{array}{l}
u=\left\langle u_{0}, u_{+}, u_{-}, u_{1}\right\rangle=\left\{u_{\beta}\right\} \\
v=\left\langle v_{0}, v_{+}, v_{-}, v_{1}\right\rangle=\left\{v_{\beta}\right\}
\end{array}\right. \\
\quad \beta \in\{0,+,-, 1\} \\
\left\{\begin{array}{l}
u_{0}=\rho_{-} \oplus \rho_{+} \\
v_{0}=\left(1-\rho_{-}\right) / 2 \oplus\left(1+\rho_{+}\right) / 2 \\
u_{+}=\rho_{+} \\
v_{+}=\left(1+\rho_{+}\right) / 2 \\
u_{-}=\rho_{-} \\
v_{-}=\left(1-\rho_{-}\right) / 2 \\
u_{1}=\rho_{-}+\rho_{+} \\
v_{1}=\left(1-\rho_{-}+\rho_{+}\right) / 2
\end{array}\right.
\end{gathered}
$$


where $0 \leq u_{\beta}, v_{\beta} \leq 1, \beta \in\{0,+,-, 1\}, \oplus$ : Asynchronous addition, + : Synchronous addition.

Using $\{u, v\}$ signals, each $u_{\beta}\left(\mathrm{v}_{\beta}\right)$ determines a fixed position in the relevant histogram to make vector $X$ on a position. After complete $2^{N}$ data sequences, eight symmetry/anti-symmetry histograms of $\left\{H\left(u_{\beta} \mid f\right)\right\}\left(\left\{H\left(v_{\beta} \mid f\right)\right\}\right) \beta \in\{0,+,-, 1\}$ are generated.

\section{Simulation Results}

The simulation provides a series of output results. In this section, two cases are selected: $N=\{12,13\}, n=2, j=0,\left\{f=3, f_{+}=11, f_{-}=2\right\}$, and $\left\{f=12, f_{+}=14, f_{-}=8\right\}$. Corresponding to double path, right path, left path, symmetric and nonsymmetric conditions, respectively. For the convenience of comparison, sample cases are shown in Fig. 3a-c. In Fig. 3a, representation patterns are illustrated. Figure $3 \mathrm{~b}$ represents $f=3$ conditions and Fig. $3 \mathrm{c}$ represents $f=12$ conditions, respectively. Eight histograms of $H\left(u_{+} \mid f\right)=H\left(u_{-} \mid f\right)$ are shown with results represented by symmetric meta-functions in four groups.

\section{Analysis of Results}

\subsection{Visual Distributions}

In $H\left(u_{+} \mid f\right)=H\left(u_{-} \mid f\right)$ conditions, $\left\{H\left(u_{1} \mid f\right), H\left(v_{1} \mid f\right)\right\}$ have significant interference patterns different from other conditions. Output results are balanced.

\subsection{Particle Statistical Distributions}

For all symmetric or nonsymmetric cases under $\oplus$ asynchronous addition operations, relevant values meet $0 \leq u_{0}, v_{0}, u_{-}, v_{-}, u_{+}, v_{+} \leq 1$.

Checking $\quad\left\{H\left(u_{0} \mid f\right), H\left(v_{0} \mid f\right)\right\} \quad$ series, $\left\{H\left(u_{+} \mid f\right), H\left(u_{-} \mid f\right)\right\} \quad$ and $\left\{H\left(v_{+} \mid f\right), H\left(v_{-} \mid f\right)\right\}$ satisfy the following equation:

$$
\left\{\begin{array}{l}
H\left(u_{0} \mid f\right)=H\left(u_{-} \mid f\right)+H\left(u_{+} \mid f\right) \\
H\left(v_{0} \mid f\right)=H\left(v_{-} \mid f\right)+H\left(v_{+} \mid f\right)
\end{array}\right.
$$

The equation is true even $N$ and $n$ in different values. 


\begin{tabular}{|c|c|c|c|c|c|}
\hline $\mathrm{N}$ & Left Path & Right Path & Double-Particle & Double-Wave & Conditions \\
\hline 12 & $H\left(u_{+} \mid f\right)$ & $H\left(u_{-} \mid f\right)$ & $H\left(u_{0} \mid f\right)$ & $H\left(u_{1} \mid f\right)$ & \multirow{2}{*}{$\begin{array}{c}\text { Symmetric Meta } \\
\text { Distributions } \\
H\left(u_{+} \mid f\right)=H\left(u_{-} \mid f\right)\end{array}$} \\
\hline 13 & $H\left(u_{+} \mid f\right)$ & $H\left(u_{-} \mid f\right)$ & $H\left(u_{0} \mid f\right)$ & $H\left(u_{1} \mid f\right)$ & \\
\hline 12 & $H\left(v_{+} \mid f\right)$ & $H\left(v_{-} \mid f\right)$ & $H\left(v_{0} \mid f\right)$ & $H\left(v_{1} \mid f\right)$ & \multirow{2}{*}{$\begin{array}{l}\text { Anti-symmetric Meta } \\
\quad \text { Distributions } \\
H\left(v_{+} \mid f\right)=H\left(1-v_{-} \mid f\right)\end{array}$} \\
\hline 13 & $H\left(v_{+} \mid f\right)$ & $H\left(v_{-} \mid f\right)$ & $H\left(v_{0} \mid f\right)$ & $H\left(v_{1} \mid f\right)$ & \\
\hline
\end{tabular}

(a) Statistical Histogram Patterns
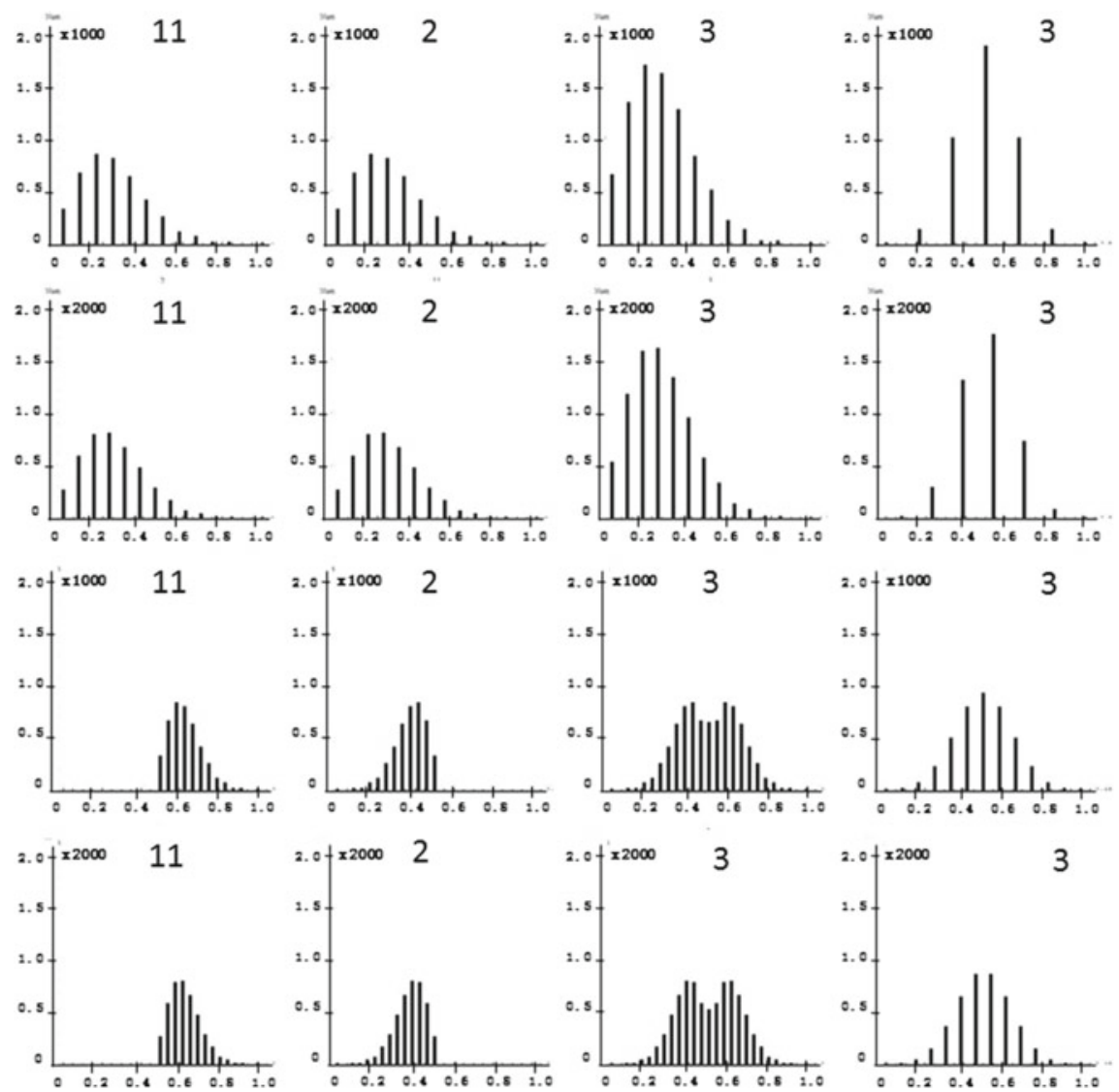

(b) $N=\{12,13\}, f=3$, Histograms of Symmetric Meta Distributions

Fig. 3 Results of symmetric meta distributions 

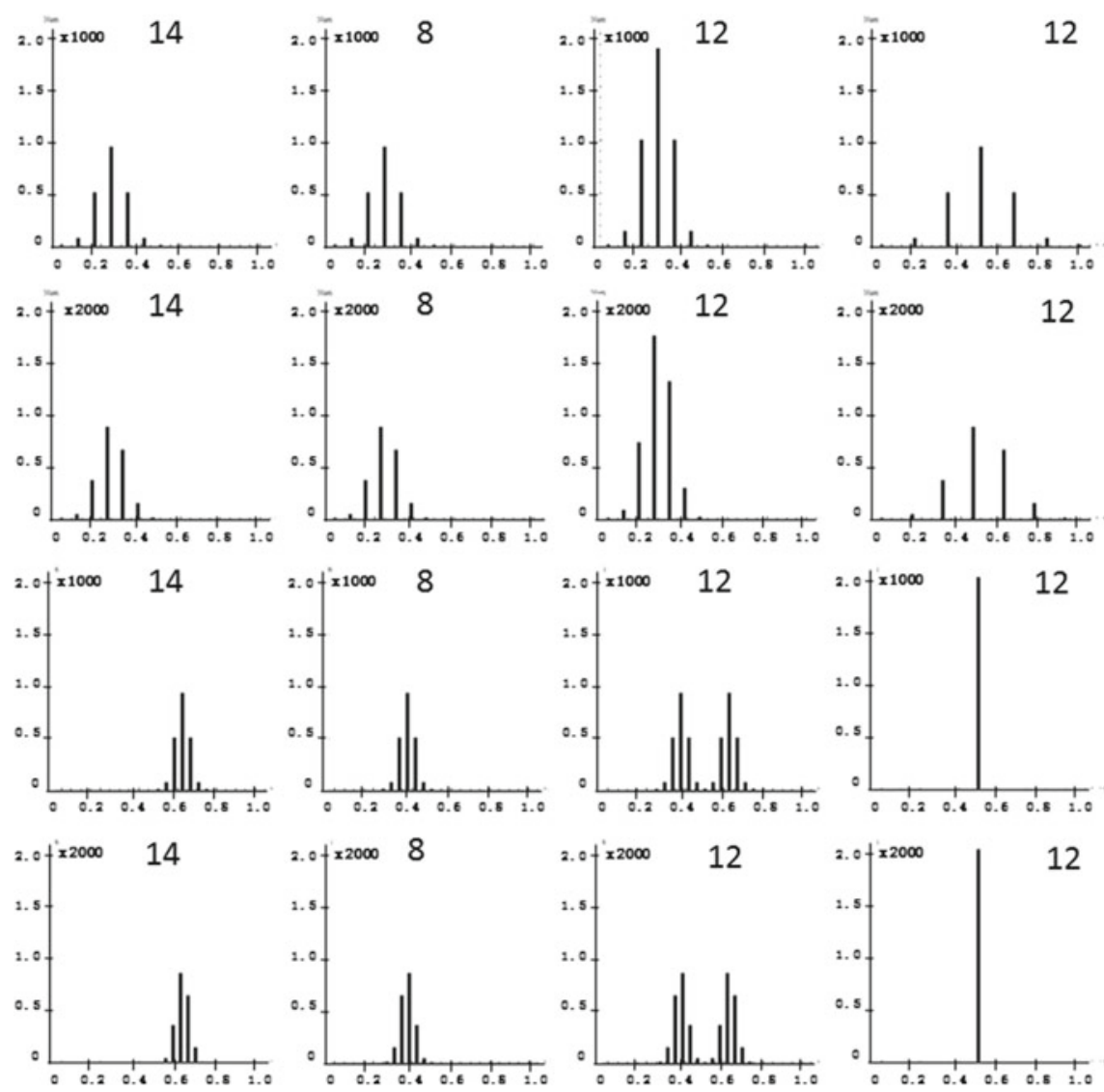

(c) $N=\{12,13\}, f=12$, Histograms of Symmetric Meta Distributions

Fig. 3 (continued)

\subsection{Wave Interference Patterns}

Different interference properties are observed clearly in $H\left(u_{+} \mid f\right)=H\left(u_{-} \mid f\right)$ and $H\left(v_{+} \mid f\right)=H\left(1-v_{-} \mid f\right)$ conditions. Under + synchronous addition operations, relevant values meet $0 \leq u_{1}, v_{1}, u_{-}, v_{-}, u_{+}, v_{+} \leq 1$.

Checking $\left\{H\left(u_{1} \mid f\right), H\left(v_{1} \mid f\right)\right\}$ distributions especially in Fig. 3b-c $\left\{u_{1}, v_{1}\right\}$ cases extremely strong interferences appeared and compared with $\left\{H\left(u_{+} \mid f\right), H\left(u_{-} \mid f\right)\right\}$ and $\left\{H\left(v_{+} \mid f\right), H\left(v_{-} \mid f\right)\right\}$, there are significant differences. Spectra in different cases illustrate wave interference properties. From listed histogram distributions, they are all satisfied 


$$
\left\{\begin{array}{c}
H\left(u_{1} \mid f\right) \neq H\left(u_{-} \mid f\right)+H\left(u_{+} \mid f\right)=H\left(u_{0} \mid f\right) \\
H\left(v_{1} \mid f\right) \neq H\left(v_{-} \mid f\right)+H\left(v_{+} \mid f\right)=H\left(v_{0} \mid f\right)
\end{array}\right.
$$

Single and double peaks are shown in interference patterns as classical double-slit distributions.

\subsection{Quaternion Measures}

It is interesting to see the relationship between the variant quaternion and other measures.

In the variant quaternion, $\Delta f(x)=\left\langle N_{\perp}, N_{+}, N_{-}, N_{T}\right\rangle, N=N_{\perp}+N_{+}+N_{-}+N_{T}$.

In Einstein's two-state system of interaction $\left\langle N_{1}, N_{2}, N_{12}, N_{21}\right\rangle$ allows the following equations to be established:

$$
\left\{\begin{array}{l}
N_{1}=N_{\perp}+N_{+} \\
N_{2}=N_{-}+N_{T} \\
N_{12}=N_{+} \\
N_{21}=N_{-} \\
N=N_{1}+N_{2}
\end{array}\right.
$$

From the equations, the measured pair $\left\{N_{21}, N_{12}\right\}$ has a 1-1 correspondence to $\left\{N_{-}, N_{+}\right\}$.

Selecting $+\rightarrow 1,-\rightarrow 0$, CHSHs $N_{ \pm, \mp}(a, b)$ measures meet

$$
\begin{gathered}
\left\{\begin{array}{l}
N_{+,+}(a, b) \rightarrow N_{T} \\
N_{+,-}(a, b) \rightarrow N_{-} \\
N_{-,+}(a, b) \rightarrow N_{+} \\
N_{-,-}(a, b) \rightarrow N_{\perp}
\end{array}\right. \\
\left(N_{++}, N_{+-}, N_{-+}, N_{--}\right) \rightarrow\left(N_{T}, N_{-}, N_{+}, N_{\perp}\right),
\end{gathered}
$$

Let $N=N_{++}+N_{+-}+N_{-+}+N_{--}$, CHSH quaternion is a permutation of the variant quaternion.

Aspect's quaternion $\left(N_{t}, N_{r}, N_{c}, N_{\omega}\right)$ have the following corresponding:

$$
\left\{\begin{array}{l}
N_{t} \rightarrow N_{-} \\
N_{r} \rightarrow N_{+} \\
N_{\omega} \rightarrow N
\end{array}\right.
$$

There is no parameter in the variant quaternion for the parameter $N_{c}$. It indicates joined action numbers to distinguish single and double paths, corresponding to 
$\left\{u_{0}, v_{0}\right\}$ and $\left\{u_{1}, v_{1}\right\}$ times. In an actual experiment, this parameter is significant. In a simulated system, the parameter is a control coefficient that separates two types of measured paths $\left\{u_{0}, v_{0}\right\}$ and $\left\{u_{1}, v_{1}\right\}$ in the integration of comparisons on real experiments.

\section{Conclusions}

Analyzing $N$ bit $0-1$ vector and its exhaustive sequences for variant measurement, this system simulates double path interference properties through different accurate distributions. Using this model, two groups of parameters $\left\{u_{\beta}\right\}$ and $\left\{v_{\beta}\right\}$ describe the left path, right path, double paths for particle, and double path for wave with distinguished symmetry and anti-symmetry properties.

Only synchronous conditions, double path system provides wave-like interference patterns different from classical ones.

Compared with the variant quaternion and other quaternion structures, it is helpful to understand possible properties of usages and limitations for variant simulation systems.

The complexity of n-variable function space has a size of $2^{2^{n}}$. Whole simulation complexity is determined by $O\left(2^{2^{n}} \times 2^{N}\right)$ as ultra exponent productions. How to overcome the limitations imposed by such complexity and how best to compare and contrast such simulations with real-world experimentation will be key issues in future work.

Acknowledgements Thanks to Mr. Colin W Campbell for making English edition, Mr. Jie Wan for generating the simulation data, and Mr. Qingping Li for making the statistical histograms.

\section{References}

1. R. Feynman, R. Leighton, M. Sands, The Feynman Lectures on Physics, vol. 3 (Addison-Wesley, Reading, MA, 1965, 1989)

2. R. Feynman, The Character of Physical Law (MIT Press, 1965)

3. J. von Neumann, Mathematical Foundations of Quantum Mechanics (Princeton University Press, 1932, 1996). ISBN 0-691-02893-1

4. J. Clauser, N. Horne, A. Shimony, R. Holt, PRL 23, 880-884 (1969)

5. A. Aspect, P. Grangier, G. Roger, Phys. Rev. Lett. 49, 91-94 (1982)

6. A. Aspect, Quantum [Un]speakables-From Bell to Quantum Information, ed. by R.A. Bertlmann, A. Zeilinger (Springer, Berlin, 2002)

7. A. Aspect, Nature 446, 866-867 (2007)

8. F. Lindner, M.G. Schätzel, H. Walther, A. Baltuska, E. Goulielmakis, F. Krausz, D.B. Milosevic, D. Bauer, W. Becker, G.G. Paulus, Phys. Rev. Lett. 95, 040401 (2005)

9. H.D. Zeh, Foundation of Physics 1, 69-76 (1970)

10. A. Zeilinger, G. Weihs, T. Jennewein, M. Aspelmeyer, Nature 433, 230-238 (2005)

11. S. Afshar et al., Found. Phys. 37, 295-305 (2007). http://www.springerlink.com/content/ q110r82074w03277/fulltext.pdf 
12. M. Arndt, O. Nairz, J. Vos-Andreae, C. Keller, G. van der Zouw, A. Zeilinger, Nature 401, 680-682 (1999)

13. S.M. Barnett, Quantum Information (Oxford University Press, Oxford, 2009). ISBN 978-019-852762-6

14. J.D. Barrow, P.C.W. Davies, J.E. Charles, L. Harper, Science and Ultimate Reality: Quantum Theory, Cosmology and Complexity (Cambridge University Press, Cambridge, 2004)

15. M. Fox, Quantum Optics (Oxford University Press, Oxford, 2006). ISBN 0-19-856672-7

16. J.C. Garrison, R. Chiao, Quantum Optics (Oxford University Press, Oxford, 2008). ISBN 978-0-850886-1

17. P. Grangier, G. Roger, A. Aspect, Europhys. Lett. 1, 173-179 (1986)

18. S. Hawking, L. Mlodinow, The Grand Design (Bantam Books, 2010)

19. R. Healey, G. Hellman (eds.), Quantum Measurement: Beyond Paradox (University of Minnesota Press, 1998). ISBN 0-8166-3065-8

20. M. Horne, A. Shimony, A. Zeilinger, Nature 347, 429-430 (1990)

21. V. Jacques et al., Science 315, 966 (2007). http://www.arxiv.org/abs/quant-ph/0610241. 13

22. M. Jammer, The Philosophy of Quantum Mechanics (Wiley-Interscience Publication, 1974). ISBN 0-471-43958-4

23. Q. Li, J. Zheng, in 11th Australian Information Warfare Conference (2010). http://ro.ecu.edu. $\mathrm{au} / \mathrm{isw} / 34$

24. P. Mittelstaedt, A. Prieur, R. Schieder, Found. Phys. 17(9), 891-903 (1987). https://doi.org/10. 1007/bf00734319

25. R. Penrose, The Road to Reality (Vintage Books, London, 2004)

26. W.P. Schleich, H. Walther (eds.), Elements of Quantum Information (Wiley-VCH Verlag GmbH \& Co KGaA Weinheim, 2007). ISBN 978-3-527-40725-5

27. N. Bohr, Discussion with Einstein on Epistemological Problems in Atomic Physics (Evanston, 1949), pp. 200-241

28. L. de Broglie, Nature 112, 540 (1923)

29. A. Einstein, Ann. Phys. 891-921 (1905)

30. A. Einstein, Mit. Phys. Ges. Zrich 16, 47 (1916)

31. J. Zheng, C. Zheng, Front. Electr. Electron. Eng. China 5, 163 (2010). http://www.springerlink. com/content/91474403127n446u/ (Higher Education Press and Springer)

32. J. Zheng, C. Zheng, T. Kunii, Cellular Automata-Innovative Modelling for Science and Engineering, ed. by A. Salcido (InTech Press, 2011). http://www.intechopen.com/articles/show/ title/a-framework-of-variant-logic-construction-for-cellular-automata

33. J. Zheng, C. Zheng, Variant measures and visualized statistical distributions. Acta Photonica Sin., to appear 2011

Open Access This chapter is licensed under the terms of the Creative Commons Attribution 4.0 International License (http://creativecommons.org/licenses/by/4.0/), which permits use, sharing, adaptation, distribution and reproduction in any medium or format, as long as you give appropriate credit to the original author(s) and the source, provide a link to the Creative Commons license and indicate if changes were made.

The images or other third party material in this chapter are included in the chapter's Creative Commons license, unless indicated otherwise in a credit line to the material. If material is not included in the chapter's Creative Commons license and your intended use is not permitted by statutory regulation or exceeds the permitted use, you will need to obtain permission directly from the copyright holder.

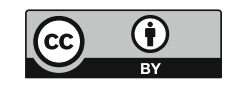

